\title{
INTERAÇÕES ENERGÉTICAS ENTRE O ESTADO DE MINAS GERAIS E O RESTANTE DO BRASIL: UMA ANÁLISE INTER-REGIONAL DE INSUMO-PRODUTO*
}

\author{
Fernando Salgueiro Perobelli ${ }^{\S}$ \\ Rogério Silva de Mattos ${ }^{\alpha}$ \\ Weslem Rodrigues Faria ${ }^{\dagger}$
}

\begin{abstract}
RESUMO
Este trabalho analisa as interações entre o Estado de Minas Gerais e o restante do Brasil com relação ao consumo de energia. A análise é feita utilizando-se um modelo inter-regional híbrido de insumo-produto, por meio do qual são computadas medidas de intensidade de uso energético. Essas medidas permitem, por exemplo, avaliar em que grau a produção de um setor de atividade dentro de Minas Gerais impacta o consumo de energia dentro e fora do Estado. Também permitem avaliar em que a produção de um setor de atividade no restante do Brasil (fora de Minas Gerais) impacta o consumo de energia dentro e fora do Estado. A análise desenvolvida apresenta informações desagregadas para 14 setores de atividade, duas áreas espaciais (Minas Gerais e restante do Brasil) e um tipo de energia consumida (energia total), permitindo traçar um retrato refinado dos padrões de interações. Conclui-se que a metodologia provê informações para o gestor/planejador estadual no desenvolvimento de estratégias eficientes, voltadas para garantir o suprimento de energia.
\end{abstract}

Palavras-chave: insumo-produto, coeficientes de requerimento, modelo inter-regional.

\begin{abstract}
This paper analyses the interactions between the State of Minas Gerais and the rest of Brazil with regard to energy consumption. A hybrid interregional input-output model, by means of which energy intensity measures are computed, is used to undertake the analysis. The energy measures allow, for instance, to assess the degree in which a sector production in Minas Gerais impacts the energy consumption inside and outside the state. Also, the measures allow to assess the degree in which a sector production in the rest of Brazil (outside Minas Gerais) impacts energy consumption inside and outside the state. The analysis presents disaggregate information for 14 economic sectors, two spatial areas (Minas Gerais and the rest of Brazil), and one kind of energy use (total energy), thus allowing to trace an accurate portrait of interdependence patterns. A conclusion is that the methodology provides relevant information for the state managers/planners in the development of efficient strategies to guarantee energy supply.
\end{abstract}

Key words: input-output, requirement coefficients, interregional model.

JEL Classification: R15, C67.

\footnotetext{
* Os autores agradecem os comentários de Alexandre Zanini, Eduardo Almeida, Fernanda Finotti, Jackson Moreira, Aryeverton Oliveira e Rafael Morais, não lhes atribuindo, porém, qualquer responsabilidade por erros e omissões associados a este artigo. Os autores também agradecem o financiamento da FAPEMIG e do CNPq para a realização desta pesquisa.

$\S$ Professor da FEA/UFJF, Coordenador do Mestrado em Economia Aplicada FEA/UFJF e Bolsista de Produtividade CNPq - Nivel 2. E-mail: fernando.perobelli@ufjf.edu.br.

a Professor da FEA/UFJF e Professor do Mestrado em Economia Aplicada FEA/UFJF. E-mail: rogerio.mattos@ufjf.edu.br.

$\dagger$ Mestrando em Economia CEDEPLAR/UFMG.E_mail: weslemrodrigues@click21.com.br.

Endereço para contato: Universidade Federal de Juiz de Fora - Faculdade de Economia e Administração - Mestrado em Economia Aplicada - Campus Universitário - Juiz de Fora - Minas Gerais - CEP: 36036-330.

Recebido em janeiro de 2005. Aprovado em fevereiro de 2007.
} 


\section{INTRODUÇÃO}

Energia é um insumo de uso generalizado na economia e, em decorrência, sua indisponibilidade pode produzir efeitos econômicos adversos de curto e de longo prazo. A crise do racionamento de energia elétrica de 2001 constitui um exemplo disso: devido a um baixo volume de chuvas, o parque gerador de energia elétrica foi insuficiente para atender à demanda prevista e, como a instalação de novas usinas requer elevados investimentos e longo prazo de maturação, o governo federal teve de introduzir um programa de racionamento. $\mathrm{O}$ efeito imediato desse racionamento foi a retração da produção e do nível de emprego (e.g., Torres e Almeida, 2003). Além disso, a escassez de energia também pode afetar o investimento agregado real da economia. Prevalecendo as incertezas sobre o suprimento adequado de energia, diversas decisões de investimento tendem a ser suspensas ou adiadas, comprometendo o crescimento econômico.

Para garantir o suprimento normal de energia, os agentes econômicos que atuam no setor de energia - órgãos de planejamento governamental, agências regulatórias e empresas fornecedoras - vêm realizando diversas ações, como intensificação das pesquisas voltadas para aumento de eficiência técnica e uso de fontes alternativas de energia; ampliação dos programas e campanhas de conservação de energia; buscas de aperfeiçoamento do aparato regulatório (como o lançamento do novo modelo regulatório para o setor elétrico em 2004 - MME, 2004) e a elaboração de um plano decenal para ampliação da capacidade de geração de energia elétrica.

No caso específico da energia elétrica, a expansão de sua oferta e, portanto, a garantia de um suprimento em quantidade e qualidade deste insumo depende, segundo Torres e Almeida (2003), de ações em diversas frentes com a participação de: a) agentes privados - que desempenham um papel fundamental no montante investido no setor e b) agentes institucionais - que são responsáveis pela definição de políticas de desenvolvimento do setor, pelas questões inerentes ao planejamento de sua expansão e pelas atividades de regulação, concessão e licenciamento ambiental.

No planejamento dessas ações, diversos estudos precisam ser feitos. Do ponto de vista dos gestores e planejadores estaduais, um aspecto de interesse diz respeito à interdependência entre produção setorial e consumo espacial de energia. A avaliação dessas interdependências por meios apropriados pode proporcionar melhor compreensão dos problemas de atendimento da demanda de energia e, conseqüentemente, melhores condições para as atividades de gestão e planejamento, no plano estadual, do suprimento de energia.

Este artigo analisa as interações, em termos setorial e regional, entre o Estado de Minas Gerais e o restante do Brasil, no que concerne ao consumo de energia. A análise é feita usando-se um modelo inter-regional híbrido de insumo-produto, que permite computar medidas de intensidade de uso energético conhecidas como requerimentos de energia. Essas medidas permitem, por exemplo, avaliar em que grau a produção de cada setor de atividade dentro de Minas Gerais impacta o consumo de energia dentro e fora do Estado. Também permitem avaliar em que grau a produção de cada setor de atividade no restante do Brasil (fora de Minas Gerais) impacta o consumo de energia dentro e fora do Estado.

Embora a análise desenvolvida neste trabalho focalize o consumo de energia de forma agregada para as diversas fontes de energia, os resultados apresentados estão desagregados segundo 14 setores de atividade e duas áreas espaciais (Minas Gerais e restante do Brasil). Isso permitiu traçar um retrato relativamente refinado dos padrões de interação entre o Estado de Minas Gerais e o restante do Brasil no que concerne ao consumo de energia.

Além desta Introdução, o artigo está organizado da seguinte forma: a Seção 2 faz uma breve análise da estrutura de consumo de energia no Brasil e em Minas Gerais; a Seção 3 apresenta a me- 
todologia utilizada, isto é, o modelo inter-regional de insumo-produto construído a partir de uma tabela híbrida de insumo-produto e a extensão desse modelo para incorporação do setor de energia; a Seção 4 descreve a base de dados utilizada; a Seção 5 apresenta e discute os resultados empíricos obtidos; a Seção 6 fecha o trabalho com algumas considerações finais.

\section{O CONSUMO DE ENERGIA NO BRASIL E EM MiNAS GERAIS}

As Tabelas 1 e 2 apresentam dados de evolução recente do consumo de energia no Brasil e em Minas Gerais, respectivamente. Os dados para o Brasil, disponíveis até 2004, foram extraídos do Balanço Energético Nacional (MME, 2005) e os de Minas Gerais, disponíveis até 2003, do Balanço Energético de Minas Gerais (CEMIG, 2005). Os números estão apresentados em uma mesma unidade, isto é, em toneladas equivalentes de petróleo (tep), para viabilizar adições e comparações. Tanto em Minas Gerais quanto no Brasil, o consumo de energia vem aumentando nos últimos anos, respondendo ao crescimento da economia como um todo.

O consumo nacional de energia em 2004, reportado na Tabela 1, chegou a cerca de 190 milhões de tep. As informações por fonte de energia indicam que esse consumo se concentra em óleo diesel (32,7 milhões) e eletricidade (30,9 milhões de tep), que, juntos, respondem por um terço do total. As informações por setor mostram que o setor de transportes (51,4 milhões) é o principal consumidor de energia no país, absorvendo cerca de $27 \%$ da energia consumida. Em seguida, vem o consumo residencial (21,4 milhões) e os setores de ferro-gusa + aço (17,9 milhões) e alimentos + bebidas (17,6 milhões).

Tabela 1 - Evolução do consumo final de energia no Brasil: 2002-2004

\begin{tabular}{lccc}
\hline Ano & 2002 & 2003 & 2004 \\
\hline $\begin{array}{l}\text { Consumo Total } \\
\text { Por Fonte }\end{array}$ & 178.160 & 182.114 & 191.128 \\
Óleo diese' & & & \\
$\quad$ Eletricidade & 31.521 & 30.885 & 32.657 \\
Bagaço de cana & 27.884 & 29.430 & 30.923 \\
Lenha & 17.495 & 19.355 & 20.273 \\
$\quad$ Outros & 14.471 & 15.218 & 15.752 \\
Por Setor & 86.789 & 87.226 & 91.523 \\
Transportes & & & \\
Residencial & 49.163 & 48.160 & 51.432 \\
Alimentos e bebidas & 20.681 & 20.902 & 21.357 \\
Ferro-gusa e aço & 15.839 & 16.659 & 17.599 \\
$\quad$ Outros & 15.729 & 16.701 & 17.945 \\
\hline
\end{tabular}

Obs: unidade - mil tep.

Fonte: BEN (MME, 2005).

Os dados para o ano de 2003 reportados na Tabela 2, indicam que o Estado de Minas Gerais responde por cerca de $13 \%$ (23,6 milhões) do consumo total de energia do país. A estrutura de consumo por fonte no Estado é similar à do Brasil: há uma concentração em fontes energéticas como óleo diesel (3,7 milhões) e eletricidade (3,6 milhões), que juntas atendem a $30 \%$ do consumo do Estado. Duas outras fontes importantes são lenha (3,1 milhões) e carvão vegetal (3,8 milhões). A estrutura de consumo por setor, no entanto, apresenta a peculiaridade de ser fortemente concen- 
trada no setor siderúrgico, com a produção de ferro-gusa e aço respondendo por $30 \%$ do consumo de energia do Estado. Em seguida, vem o setor de transportes ( 5 milhões) e o consumo residencial (3,5 milhões).

Tabela 2 - Evolução do consumo final de energia em Minas Gerais: 2001-2003

\begin{tabular}{lccc}
\hline Ano & 2001 & 2002 & 2003 \\
\hline $\begin{array}{l}\text { Consumo Total } \\
\text { Por Fonte }\end{array}$ & 22.381 & 23.037 & 23.596 \\
$\quad$ Óleo diesel & & & \\
$\quad$ Eletricidade & 3.750 & 3.742 & 3.742 \\
$\quad$ Lenha & 3.322 & 3.482 & 3.562 \\
$\quad$ Carvão vegetal & 3.235 & 3.200 & 3.095 \\
$\quad$ Outros & 2.775 & 2.913 & 3.771 \\
$\quad$ Por Setor & 9.299 & 9.700 & 9.426 \\
$\quad$ Transportes & & & \\
Ferro-gusa e aço integrado & 5.154 & 5.119 & 5.048 \\
$\quad$ Residencial & 4.351 & 4.609 & 4.627 \\
$\quad$ Ferro-gusa não integrado & 3.735 & 3.688 & 3.541 \\
$\quad$ Outros & 1.891 & 1.911 & 2.677 \\
\hline
\end{tabular}

Obs: unidade - mil tep.

Fonte: BEEMG (CEMIG, 2005).

\section{Metodologia}

A análise de insumo-produto é freqüentemente utilizada para se estudar as interdependências ou interações entre setores da economia de uma região ou país. O grau de interdependência pode ser avaliado por meio de medidas conhecidas como coeficientes de requerimento intersetorial. Esses coeficientes permitem avaliar, por exemplo, os impactos que mudanças na demanda final de um setor exercem sobre os demais setores da economia (Miller e Blair, 1985). Existem várias extensões possíveis da análise de insumo-produto, dentre as quais, de particular relevância para este trabalho, estão a hipótese de várias regiões e a incorporação do setor energético. A primeira permite o estudo das interações setoriais entre diversas regiões (Isard et al., 1998). Por exemplo, podem ser avaliados os impactos de mudanças na demanda final de um setor sobre todos os setores da mesma região e das demais regiões consideradas. A incorporação do setor energético é de interesse porque a produção de todos os bens da economia envolve o consumo de, pelo menos, um insumo energético. Deste modo, a interdependência intersetorial e inter-regional, em termos do consumo de energia, também pode ser medida e estudada. Esta seção descreve um modelo de insumo-produto inter-regional com incorporação do setor de energia juntamente com as medidas de interdependência energética, que foram calculadas e analisadas neste trabalho.

\subsection{Modelo inter-regional de insumo-produto}

O modelo inter-regional de insumo-produto (IR-IP) descreve os fluxos monetários de bens e serviços através da economia considerando diferentes regiões. No caso mais simples (aqui considerado) de uma economia dividida em duas regiões e $n$ setores, o modelo IR-IP pode ser representado matematicamente, em notação matricial, como: 


$$
Z i_{2 n}+Y=X
$$

em que $Z=\left\{z_{i j}\right\}$ é uma matriz $2 n \times 2 n$, que representa a tabela de insumo-produto, $i_{2 n}$ é um vetor unitário (todos os seus elementos são iguais a 1) de ordem $2 n \times 1 ; Y=\left\{y_{j}\right\}$ é um vetor $2 n \times 1$ cujos elementos são as demandas finais de ambas as regiões, e $X=\left\{x_{j}\right\}$ é um vetor $2 n \times 1$, cujos elementos são as produções setoriais, também de ambas as regiões.

Uma forma mais conveniente de escrever a equação (1) é definir a matriz de coeficientes técnicos:

$$
A=Z(\hat{X})^{-1}
$$

em que $\hat{X}=\operatorname{diag}(X)$. Cada elemento de $A$ é definido, de modo geral, como $a_{i j}=x_{i j} / x_{j}$ e corresponde à proporção de insumos do setor $i$ necessária à produção de $\mathrm{R} \$ 1$ de produto do setor $j$, sendo que as regiões do setor $i$ e do setor $j$ podem ser as mesmas ou não. Assim, os elementos de $A$ são chamados de coeficientes de requerimento direto e se dividem em dois tipos: $a_{i j}^{L L}$ e $a_{i j}^{M M}$ são os coeficientes intra-regionais e $a_{i j}^{L M}$ e $a_{i j}^{M L}$ são os coeficientes inter-regionais. Isso permite que a matriz $A$ possa ser particionada em quatro submatrizes:

$$
A=\left[\begin{array}{ll}
A^{L L} & A^{L M} \\
A^{M L} & A^{M M}
\end{array}\right]
$$

em que $A^{L L}$ e $A^{M M}$ são as matrizes de coeficientes intra-regionais e $A^{L M}$ e $A^{M L}$ as matrizes de coeficientes inter-regionais. O sistema (3) pode então ser reescrito como:

$$
A X+Y=X
$$

de forma que, após breve manipulação algébrica, obtém-se:

$$
X=B Y
$$

em que $B=(I-A)^{-1}$ corresponde à matriz de Leontief para o modelo IR-IP. Subtraindo-se de $B$ a matriz identidade, obtém-se:

$$
R=B-I
$$

em que $R$ é uma matriz de coeficientes de requerimento líquido total ${ }^{l}$. Observe-se que essa matriz também pode ser particionada em quatro submatrizes:

$$
R=\left[\begin{array}{ll}
R^{L L} & R^{L M} \\
R^{M L} & R^{M M}
\end{array}\right]
$$

em que $R^{L L}$ e $R^{M M}$ são matrizes de coeficientes intra-regionais e $R^{L M}$ e $R^{M L}$ são matrizes de coeficientes inter-regionais. Também é possível definir a matriz de coeficientes de requerimento indireto:

1 A subtração da matriz identidade tem o papel de extrair da matriz B os requerimentos iniciais produzidos pela demanda final de cada setor. Desta forma, são eliminados certos problemas de comparação dos requerimentos diretos e indiretos entre os setores, - ver Miller e Blair (1985). 


$$
Q=R-A=\left[\begin{array}{ll}
Q^{L L} & Q^{L M} \\
Q^{M L} & Q^{M M}
\end{array}\right]
$$

em que $Q^{L L}$ e $Q^{M M}$ são matrizes de coeficientes intra-regionais e $Q^{L M}$ e $Q^{M L}$ são matrizes de coeficientes inter-regionais.

As matrizes $A, R$ e $Q$ provêem informações numéricas sobre o grau de dependência/interação direta, total e indireta das atividades produtivas entre setores e regiões, respectivamente. Os coeficientes da matriz $A$ - de requerimentos diretos de energia - provêem informações sobre os efeitos de primeira ordem, enquanto a matriz $Q-$ de requerimentos indiretos de energia - capta os efeitos de ordens superiores decorrentes de uma mudança da demanda final.

\subsection{Incorporação do setor de energia}

Esta seção apresenta uma extensão do modelo inter-regional de insumo-produto para incorporação do setor de energia. Existem diferentes formas de se fazer essa extensão e a abordagem utilizada neste trabalho se baseia na construção de uma tabela híbrida de insumo-produto. Essa abordagem foi usada por vários autores, como Miller e Blair (1985), Gowdy e Miller (1987), Machado (2002) e Hilgemberg (2004).

A idéia básica é incluir uma linha e uma coluna (para cada região) representando o setor energético na tabela de insumo-produto $Z$. A linha corresponde às vendas do setor de energia para os outros setores, com a característica de que essas vendas são medidas em unidades físicas. A coluna representa as compras do setor de energia aos outros setores com essas compras medidas em unidades monetárias. Assim, essa tabela expandida de insumo-produto apresentará fluxos econômicos expressos em unidades híbridas, ou seja, com algumas transações intersetoriais representadas em valores monetários e outras em unidades físicas.

Segundo Bullard e Herendeen (1975), Miller e Blair (1985) e Casler e Blair (1997), o modelo de insumo-produto em unidades híbridas é a formulação mais consistente para aplicação de modelos de insumo-produto de natureza físico-econômica envolvendo uso de energia. ${ }^{2}$

Hawdon e Pearson (1995) e Zhang e Folmer (1998) apontam algumas vantagens do uso da estrutura de insumo-produto para analisar questões relativas ao setor energético: a) permite uma desagregação setorial maior do que os modelos de otimização dinâmica e os modelos macroeconômicos; b) permite a incorporação de fluxos de energia intersetoriais tanto em termos físicos quanto monetários, e c) possibilita implementar análises de impacto. Entretanto, esses modelos também apresentam algumas limitações, quais sejam: a) coeficientes fixos de insumo-produto; b) retornos constantes de escala, e c) demanda final determinada exogenamente. É importante salientar que tais limitações não invalidam os resultados do modelo.

Considere a notação matricial em (1) reescrita da seguinte forma:

$$
Z^{*} i_{2(n+1)}+Y^{*}=X^{*}
$$

em que:

2 O modelo de insumo-produto em unidades híbridas construído por Bullard e Herendeen (1975) objetivava superar os problemas e limitações apresentados ao modelo de impactos totais dos coeficientes diretos sobre a matriz inversa de Leontief. 


$$
Z^{*}=\left[\begin{array}{cc}
Z^{L L} & Z^{L M} \\
e^{L L} & e^{L M} \\
Z^{M L} & Z^{M M} \\
e^{M L} & e^{M M}
\end{array}\right], Y^{*}=\left[\begin{array}{c}
Y^{L} \\
e_{Y}^{L} \\
Y^{M} \\
e_{Y}^{M}
\end{array}\right] \text { e } X^{*}=\left[\begin{array}{c}
X^{L} \\
e_{X}^{L} \\
X^{M} \\
e_{X}^{M}
\end{array}\right]
$$

Note-se que $Z^{*}$ representa uma tabela de insumo-produto híbrida. As submatrizes $Z^{L L}$ e $Z^{M M}$, referentes às transações setoriais intra-regionais, e as submatrizes $Z^{L M}$ e $Z^{M L}$, referentes às transações setoriais inter-regionais, apresentam elementos medidos em unidades monetárias; por sua vez, os vetores $e^{L L}$ e $e^{M M}$, referentes às transações setoriais intra-regionais de energia, e os vetores $e^{L M} \mathrm{e}$ $e^{M L}$, referentes às transações setoriais inter-regionais de energia, são medidos em unidades físicas. Os componentes $Y^{L}, Y^{M}, X^{L}$ e $X^{M}$ são vetores $n \times 1$, contendo as demandas finais e os produtos setoriais, respectivamente, nas regiões $L$ e $M$.

Definindo $\hat{X}^{*}=\operatorname{diag}\left(X^{*}\right)$, é possível construir uma matriz híbrida de coeficientes de requerimento direto como:

$$
A^{*}=Z^{*}\left(\hat{X}^{*}\right)^{-1}
$$

o que, por sua vez, permite reescrever (8) como:

$$
A^{*} X^{*}+Y^{*}=X^{*}
$$

Após breve manipulação algébrica, obtém-se:

$$
X^{*}=B^{*} Y^{*}
$$

em que $B^{*}=\left(I-A^{*}\right)^{-1}$. É possível, então, construir uma matriz híbrida de coeficientes de requerimento líquido total como:

$$
R^{*}=B^{*}-I^{*}
$$

em que $I^{*}$ é a matriz identidade $2(n+1) \times 2(n+1)$.

Neste trabalho, o interesse está centrado na estrutura de dependência intra e inter-regional em termos do setor energético, visando extrair tanto da matriz $A^{*}$ como da matriz $R^{*}$ apenas as informações de requerimento relativas ao setor de energia. Para isso, define-se, primeiramente, a seguinte matriz composta apenas por zeros e uns:

$$
\begin{aligned}
& U=\left[\begin{array}{cc}
\mathbf{0}^{L L} & \mathbf{0}^{L M} \\
i_{n+1}^{\prime} & i_{n+1}^{\prime} \\
\mathbf{0}^{M L} & \mathbf{0}^{M M} \\
i_{n+1}^{\prime} & i_{n+1}^{\prime}
\end{array}\right] \\
& V=\left[\begin{array}{cc}
i_{n+1}^{\prime} & \mathbf{0} \\
\mathbf{0} & i_{n+1}^{\prime}
\end{array}\right]
\end{aligned}
$$


Na matriz $U$, as submatrizes '0', de elementos nulos, têm o papel de eliminar dos cômputos posteriores as informações de requerimento dos setores não energéticos em ambas as regiões; por sua vez, o elemento $i_{n+1}$ representa um vetor unitário de ordem $(n+1) \times 1$ e a presença de quatro desses vetores na matriz $U$ tem o papel de incorporar as informações de requerimentos do setor energético nos cômputos posteriores. A matriz $V$ é usada apenas para compactar as matrizes de resultados.

É possível, então, calcular os requerimentos intra e inter-regionais de energia por setor, conforme segue:

$$
\begin{aligned}
& \Pi=V\left(U \circ A^{*}\right) \\
& \Lambda=V\left(U \circ R^{*}\right) \\
& \mathrm{P}=\Lambda-\Pi
\end{aligned}
$$

Nas expressões acima, o símbolo 'o’ representa produto matricial elemento a elemento. $\Pi, \Lambda$ e $\mathrm{P}$ são matrizes $2 \times 2(n+1)$ que representam, respectivamente, requerimentos diretos, totais e indiretos de energia. Cada uma dessas matrizes pode ser particionada em quatro subvetores de ordem $1 \times(n+1)$, representando os requerimentos intra e inter-regionais:

$$
\begin{aligned}
& \Pi=\left[\begin{array}{ll}
\pi^{L L} & \pi^{L M} \\
\pi^{M L} & \pi^{M M}
\end{array}\right] \\
& \Lambda=\left[\begin{array}{ll}
\lambda^{L L} & \lambda^{L M} \\
\lambda^{M L} & \lambda^{M M}
\end{array}\right] \\
& \mathbf{P}=\left[\begin{array}{ll}
\rho^{L L} & \rho^{L M} \\
\rho^{M L} & \rho^{M M}
\end{array}\right]
\end{aligned}
$$

Assim, as matrizes $\Pi, \Lambda$ e $\mathrm{P}$ provêem informações numéricas sobre a estrutura de dependência/interação direta, total e indireta, respectivamente, e de forma intra e inter-regional, existente entre os diversos setores econômicos com o setor de energia em particular.

\section{BASE DE DADOS}

A base de dados necessária para implementação do modelo de insumo-produto em unidades híbridas é formada por dois tipos de dados: a) matriz de insumo-produto e b) balanço energético. Este trabalho faz uso da matriz inter-regional de insumo-produto de Minas Gerais $\times$ restante do Brasil, calculada com base no ano de 1996 (BDMG e FIPE, 2002); dos dados de uso setorial de energia disponíveis no Balanço Energético Nacional de 2005, com base no ano de 2004 (MME, 2005), e dos dados de uso setorial de energia disponíveis no $19^{\circ}$ Balanço Energético do Estado de Minas Gerais (BEEMG), com base no ano de 2003 (CEMIG, 2005). 
Como a agregação setorial da matriz de Minas Gerais $\times$ restante do Brasil é diferente da estrutura setorial apresentada nos balanços energéticos de ambas regiões, foi necessário fazer uma compatibilização setorial dessas distintas bases de dados. A matriz de Minas Gerais $\times$ restante do Brasil está desagregada para 40 setores, ao passo que o balanço energético, tanto nacional quanto de Minas Gerais, fornece dados desagregados de consumo de energia para apenas 20 setores. A compatibilização levou a uma matriz com 14 setores (ver Anexo).

\section{ANÁlise dos RESULTADOS}

O modelo de IR-IP, descrito na Seção 3, permite computar informações bastante desagregadas sobre os padrões setoriais de interação energética entre Minas Gerais e o restante do Brasil. Podem ser computados, para cada região, os requerimentos totais intra e inter-regionais, que podem esses ser divididos em diretos e indiretos. A seqüência dos procedimentos de desagregação ainda pode ser feita de diferentes maneiras, segundo o interesse particular da análise que se deseja fazer.

Nesta seção, apresenta-se uma análise apenas para o comportamento dos requerimentos totais de energia (matriz $\Lambda$ da equação 18), considerando a desagregação desses requerimentos em intra e inter-regionais e, posteriormente, em seus respectivos componentes direto e indireto. O quadro obtido, embora parcial, proporciona uma visão útil das interações setoriais/espaciais do consumo de energia e ilustra a potencialidade da metodologia para análises mais desagregadas e extensas.

Para melhor visualização, os resultados numéricos são apresentados em gráficos de barras, ao invés de tabelas, e são analisados em duas partes. Na primeira, são estudados os requerimentos líquidos totais por setor e sua decomposição em requerimentos intra e inter-regionais para Minas Gerais e restante do Brasil. Na segunda, os vetores de requerimento setorial intra e inter-regionais são estudados em termos de sua divisão em requerimentos diretos e indiretos. Um aspecto a ser observado é que os requerimentos inter-regionais de energia são considerados sob a ótica das vendas: isto é, o requerimento inter-regional de Minas Gerais refere-se às vendas de energia feitas por esse Estado ao restante do Brasil; por sua vez, o requerimento inter-regional do restante do Brasil referese às vendas de energia feitas por essa região ao Estado de Minas Gerais.

\subsection{Requerimentos líquidos totais}

Os Gráficos 1 e 2 apresentam os valores numéricos dos requerimentos líquidos totais absolutos intra e inter-regionais para 14 setores de atividade. No Gráfico 1, referente a Minas Gerais, os requerimentos intra-regionais indicam um montante destacado para o setor Ferro + Aço $(0,38)$, que vem seguido pelos setores Transporte $(0,22)$, Energético $(0,18)$ e Outras Indústrias $(0,14)$. Os requerimentos inter-regionais (pressão do restante do Brasil sobre Minas Gerais), por sua vez, apresentam patamares bem mais baixos, com destaque para os setores Energético $(0,16)$, Transporte $(0,10)$, Outras Indústrias $(0,10)$ e Alimentos + Bebidas $(0,10)$.

No caso dos requerimentos intra-regionais do restante do Brasil, o Gráfico 2 apresenta, em destaque, o setor de Transportes (0,44), seguido pelos setores Energético (0,27), Alimentos + Bebidas $(0,21)$, Outras Indústrias $(0,18)$ e Comércio + Serviços $(0,14)$. Os requerimentos inter-regionais (pressão de Minas Gerais sobre o restante do Brasil), por sua vez, apresentam patamares muito pequenos vis-à-vis os inter-regionais, com algum destaque aparecendo apenas para o setor de Ferro e Aço $(0,05)$. 
Gráfico 1 - Requerimento total líquido de energia do setor energético de Minas Gerais

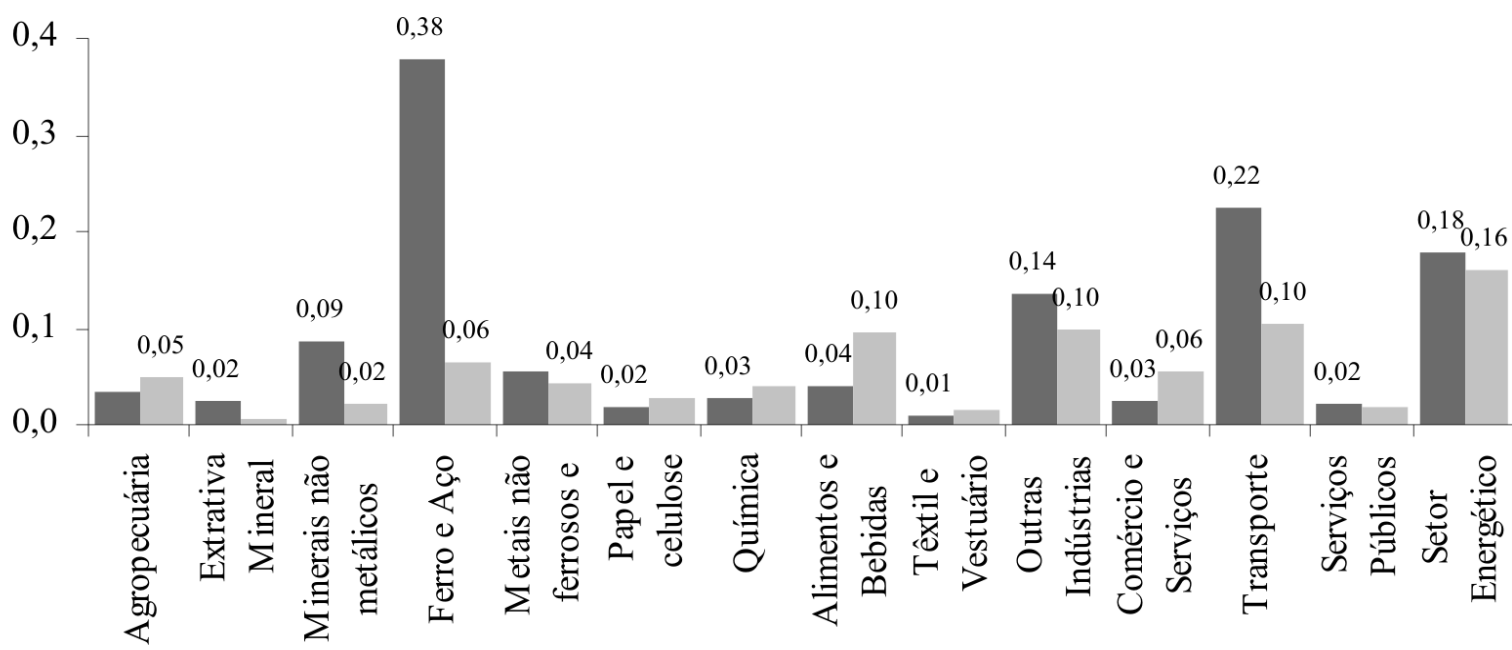

\section{Intra Inter}

Fonte: dados da pesquisa.

Gráfico 2 - Requerimento total líquido de energia do setor energético do restante do Brasil

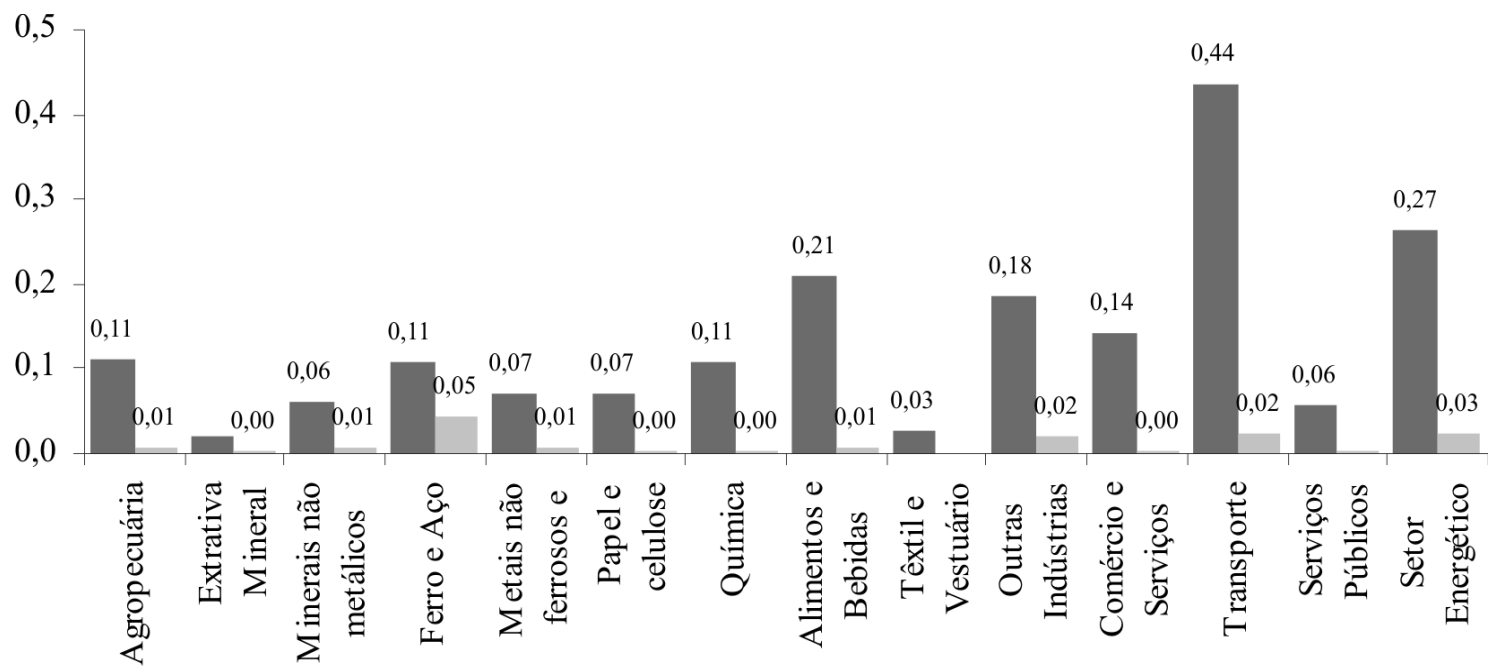

Intra Inter

Fonte: dados da pesquisa. 


\subsection{Decomposição regional dos requerimentos líquidos}

Os Gráficos 3 e 4 apresentam a decomposição porcentual dos requerimentos líquidos totais em seus componentes intra e inter-regional. Em Minas Gerais, os efeitos inter-regionais possuem um peso mais expressivo por setor do que no caso do restante do Brasil. Naquele Estado, o menor porcentual de requerimento inter-regional ocorre para o setor Ferro e Aço (14,5\%) e o maior, por sua vez, ocorre para o setor Alimentos + Bebidas (70,2\%). No restante do Brasil, os porcentuais de efeitos inter-regionais variam de 3,1\%, para os setores Papel + Celulose e Comércio+Serviços, até um máximo de 29,2\%, para o setor Ferro e Aço.

Esse aspecto indica, naturalmente, uma maior influência do restante do Brasil pressionando o setor de energia em Minas Gerais do que o contrário. Mas é valido observar no Gráfico 3, que o peso de Minas Gerais (requerimentos inter-regionais) na demanda sobre o setor de energia do restante do Brasil não é desprezível, sobretudo ao se considerar que existem outros 26 Estados na Federação.

Gráfico 3 - Participação intra e inter-regional no requerimento líquido total de energia em Minas Gerais

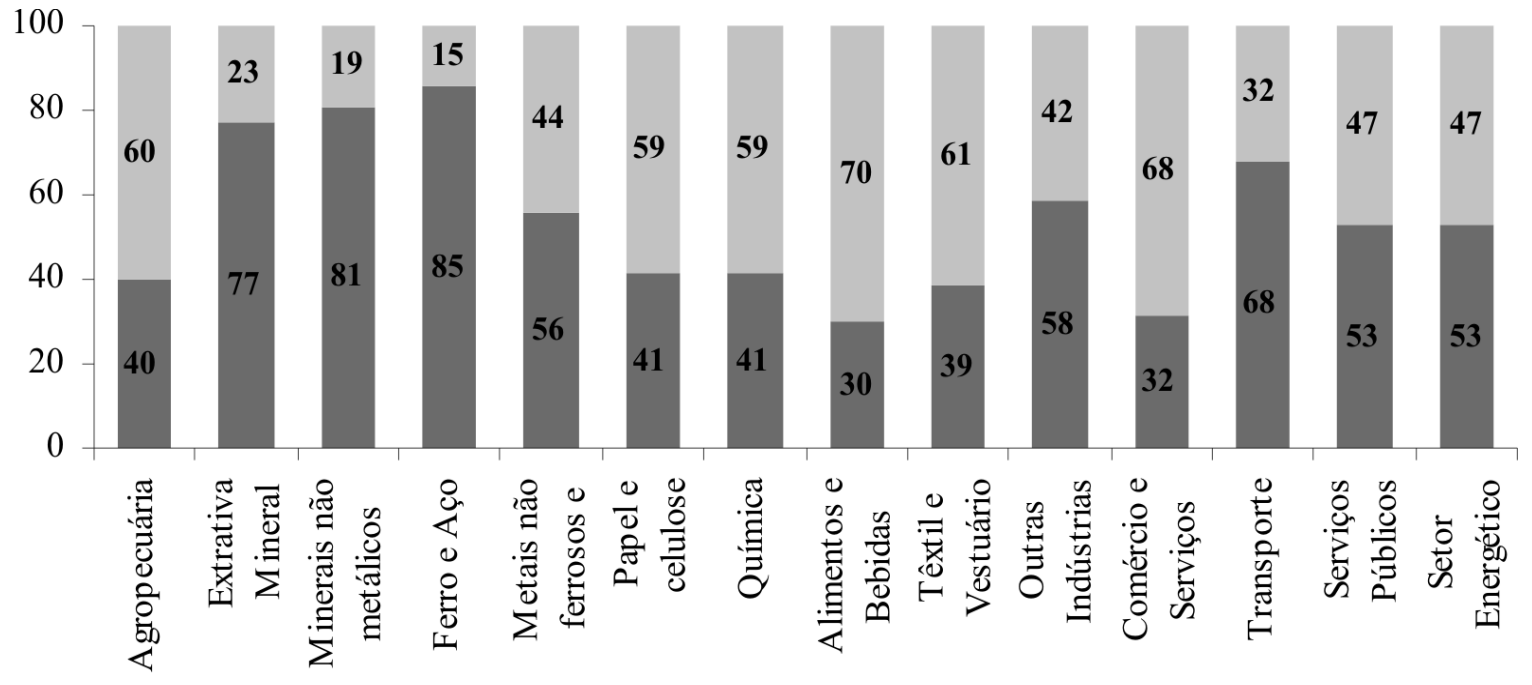

Intra $\square$ Inter

Fonte: dados da pesquisa. 
Gráfico 4 - Participação intra e inter-regional no requerimento líquido total de energia no restante do Brasil

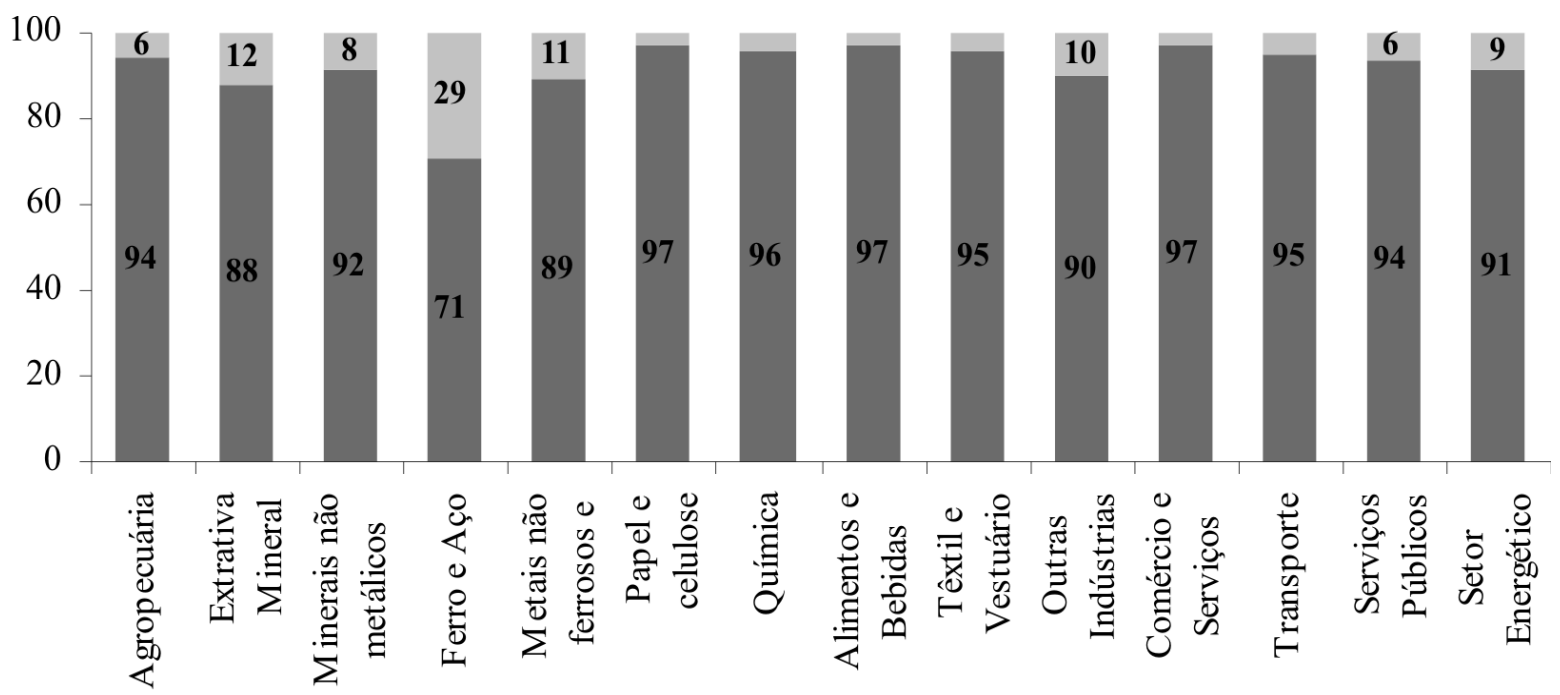

Intra $\square$ Inter

Fonte: dados da pesquisa.

\subsection{Componentes direto e indireto dos requerimentos}

Esta subseção analisa a composição dos requerimentos regionais (intra ou inter) em termos de efeitos diretos e indiretos produzidos sobre os setores de energia das regiões estudadas. Nos resultados apresentados a seguir, deve-se observar que, quanto menor a relação requerimentos diretos versus indiretos, maior o poder de multiplicação que a atividade de um dado setor exerce sobre o consumo de energia dentro de uma região. Setores com alto peso na demanda de energia da região e que, ao mesmo tempo, apresentam uma baixa relação requerimentos diretos versus indiretos tendem a produzir as mais fortes pressões de demanda sobre o setor de energia da região. Em um outro extremo, estariam setores com baixo peso na demanda de energia da região e com alta relação requerimentos diretos versus indiretos, que, neste caso, produziriam pequenas pressões sobre o setor de energia da região. Entre ambos os extremos, estão setores com graus variados, intermediários de importância na pressão que exercem. Essa perspectiva se aplica tanto quando se foca os requerimentos intra como os inter-regionais.

Os Gráficos 5 e 6 apresentam a decomposição porcentual dos requerimentos intra-regionais em seus componentes direto e indireto. A estrutura setorial dos requerimentos em termos da composição direto-indireto se mostra bastante diversificada por setor tanto em Minas Gerais como no restante do Brasil. Para Minas Gerais, há um setor com participação dos efeitos diretos abaixo de $6 \%$ (Outras Indústrias) e três setores com participação acima de 70\% (Minerais Não-Metálicos, Papel + Celulose e Transporte). Note-se que, para esse Estado, o setor Outras Indústrias, que tem peso significativo no consumo total de energia do Estado, apresenta uma baixíssima relação requerimentos diretos versus indiretos, indicando que exerce forte pressão sobre o setor de energia de Minas Gerais. 
Gráfico 5 - Minas Gerais: participação porcentual setorial no requerimento líquido total intraregional

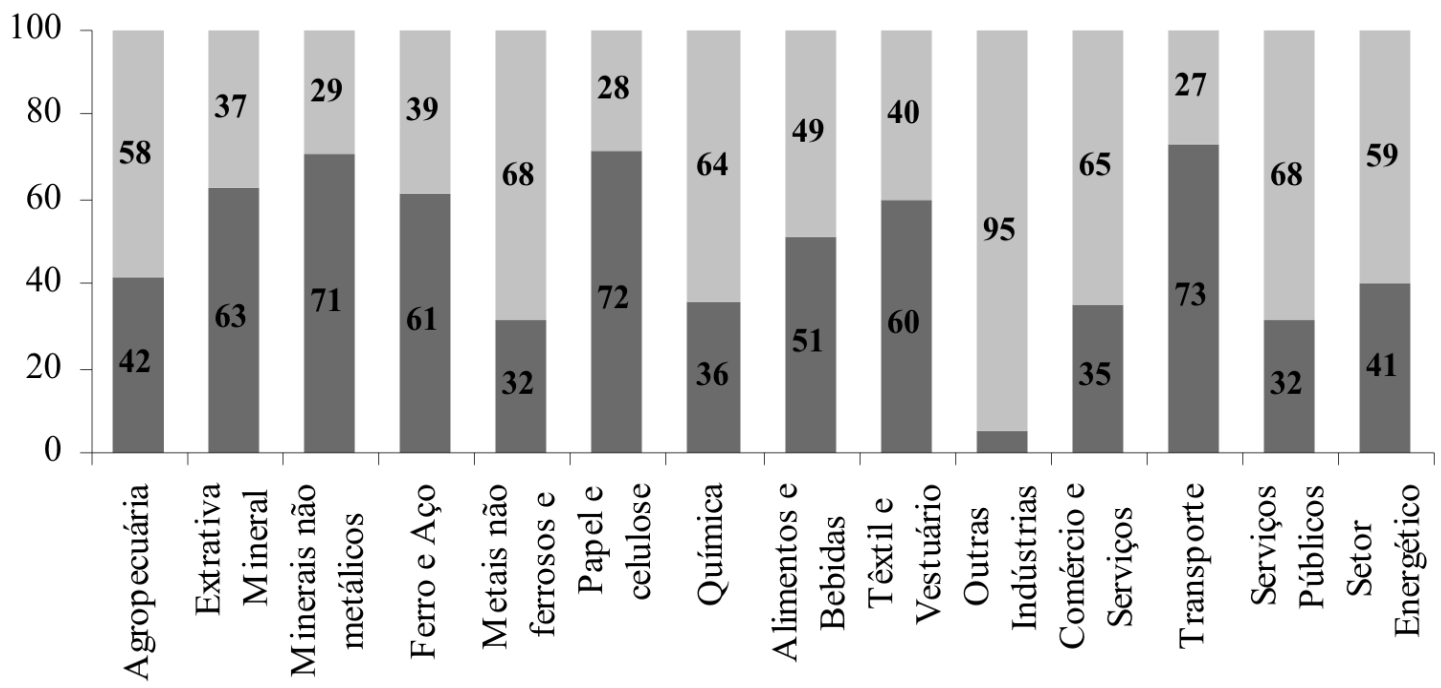

$\square$ Direto Indireto

Fonte: dados da pesquisa.

Gráfico 6 - Restante do Brasil: participação porcentual setorial no requerimento líquido total intra-regional

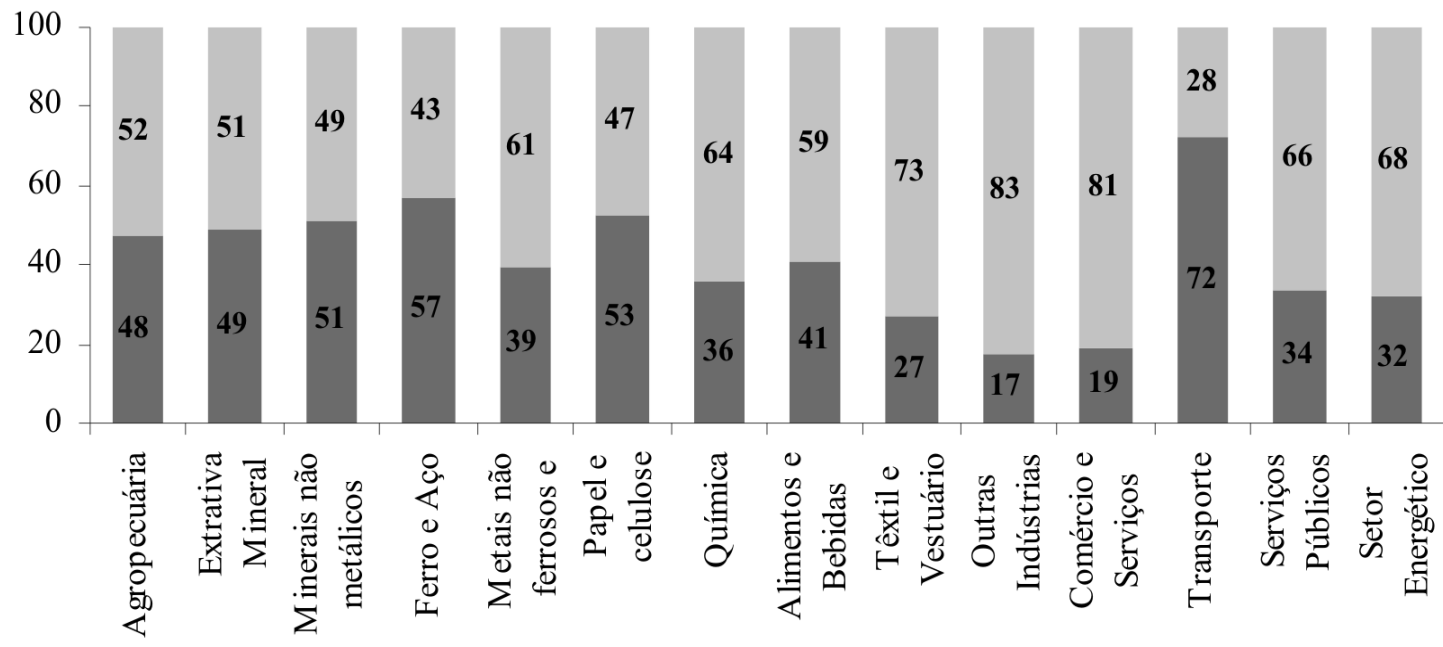

Direto Indireto

Fonte: dados da pesquisa. 
Para o restante do Brasil, há dois setores com participação dos requerimentos intra-regionais diretos abaixo de 20\% (Outras Indústrias e Comércio + Serviços) e apenas um setor com participação acima de $70 \%$ (Transporte). Aqui também se observa uma baixa relação requerimentos diretos versus indiretos para o setor Outras Indústrias, que apresenta um peso elevado no consumo total de energia do restante do Brasil e, logo, exerce forte pressão sobre o setor de energia dessa região.

Os Quadros 7 e 8 apresentam, por sua vez, a decomposição porcentual dos requerimentos inter-regionais em seus componentes direto e indireto. Note-se que os porcentuais de requerimentos diretos inter-regionais, em média, são menores do que os efeitos intra-regionais (Gráficos 5 e 6) e que a distribuição por setor é significativamente diferente. Note-se também que as relações requerimentos diretos versus indiretos são ainda menores (tanto para Minas Gerais quanto para o restante do Brasil), indicando um maior poder de multiplicação dos efeitos das atividades setoriais. Embora mais evidente no caso de Minas Gerais, isso também é válido no caso do restante do Brasil. No caso de Minas Gerais, destacam-se os setores Outras Indústrias (4,8\%), Comércio + Serviços (6,2\%) e Serviços Públicos (8\%).

Gráfico 7 - Minas Gerais: participação porcentual setorial no requerimento líquido total interregional

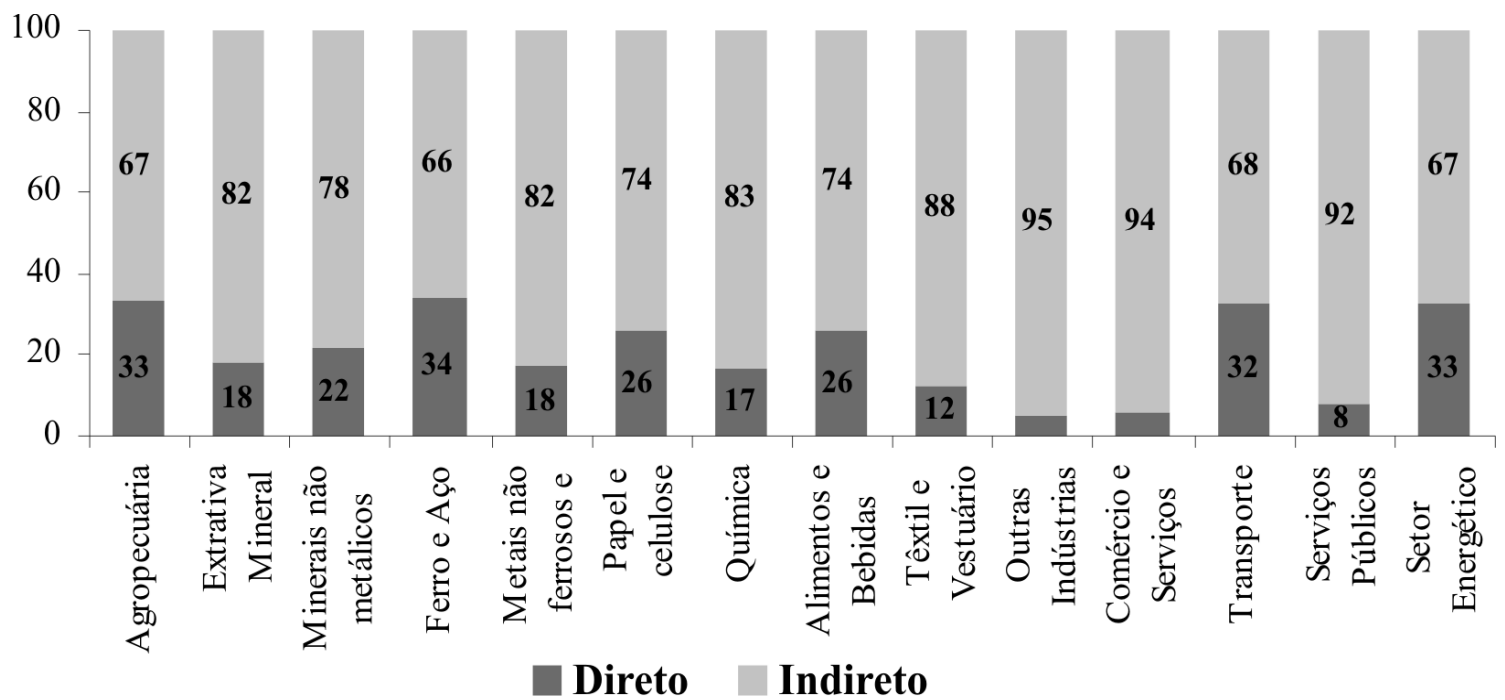

Fonte: dados da pesquisa. 


\section{Gráfico 8 - Restante do Brasil: Participação porcentual setorial no requerimento líquido total inter-regional}

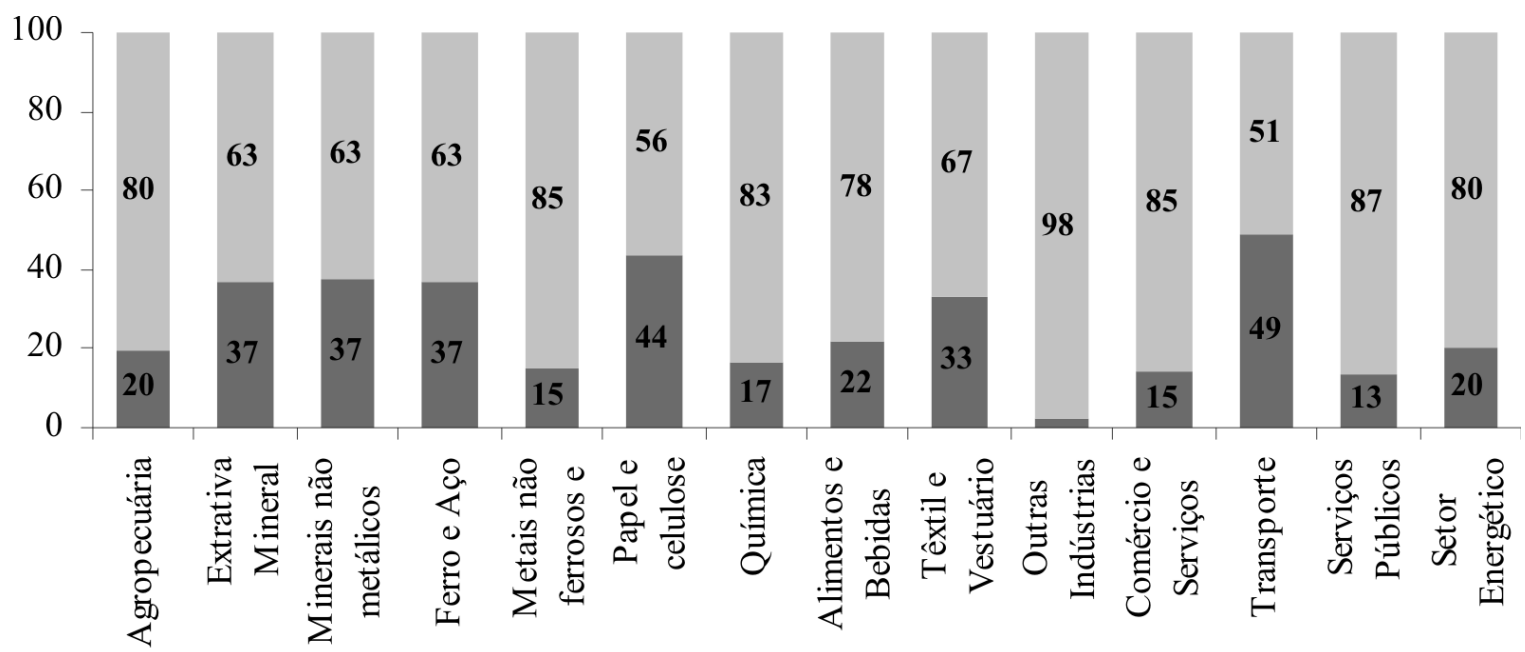

\section{$\square$ Direto Indireto}

Fonte: dados da pesquisa.

\subsection{Implicações de política e gestão}

Do ponto de vista dos gestores/planejadores do suprimento de energia, que atuam em âmbito nacional, a aplicação feita neste trabalho, com a metodologia descrita na Seção 4, é de uso limitado, porque está focada nas interações energéticas de um único Estado, no caso, o de Minas Gerais, com o restante do país. Para o gestor/planejador nacional, o ideal seria aplicar a metodologia em bloco para todos os Estados da Federação ou então para todas as principais regiões consumidoras de energia. Esse tipo de análise teria de ser feita com um modelo multirregional de insumo-produto (com várias e não apenas duas regiões) e poderia ser relevante para mostrar uma rede de complexas interações energéticas setoriais/espaciais entre várias regiões no espaço brasileiro, fornecendo, assim, melhores bases para a gestão e o planejamento nacional do suprimento de energia.

Por outro lado, sob a ótica dos gestores e planejadores estaduais em Minas Gerais, a aplicação aqui feita proporciona resultados mais imediatos. Como visto anteriormente nesta seção, foi possível avaliar as pressões sobre o setor de energia de Minas Gerais, separando aquelas que se originam de setores dentro do próprio Estado daquelas que se originam de setores fora do Estado. Na grande maioria, os setores econômicos dentro de Minas Gerais exercem maior pressão sobre o setor de energia do Estado do que os respectivos setores econômicos fora do Estado. A análise dos requerimentos intra e inter-regionais (Gráfico 1) indica que, dentro de Minas Gerais, os setores Ferro e Aço, Transporte, Energético e Outras Indústrias representam um peso significativo na demanda incidente sobre o setor de energia do Estado. Além disso, embora o setor Outras Indústrias, em particular, se mostre o último da lista dos setores importantes, a sua baixa relação requerimentos diretos versus indiretos indica que o mesmo exerce pressões potenciais mais significativas do que aparenta sobre o setor de energia no Estado (Gráfico 5).

Por sua vez, os setores situados fora de Minas Gerais que possuem maior peso na demanda sobre o setor de energia desse Estado são: Energético, Transporte, Outras Indústrias e Alimentos + Bebidas (Gráfico 1). Deve-se observar que esses setores ainda apresentam uma baixa relação 
requerimentos diretos versus indiretos, sobretudo o setor Outras Indústrias (Gráfico 7), e, desta forma, possuem alto poder de multiplicação de suas atividades sobre o setor de energia de Minas Gerais.

Essas informações trazem relevantes implicações para a gestão e o planejamento estaduais, voltados para o suprimento de energia no Estado (e pelo Estado). Primeiro, elas indicam que um acompanhamento cuidadoso do comportamento dinâmico desses setores dentro e fora de Minas Gerais (identificados como maiores demandantes do setor de energia do Estado) é relevante como estratégia de prevenção contra problemas de abastecimento de energia no curto prazo. Em segundo lugar, elas indicam também onde concentrar os esforços, tanto na formulação de políticas setoriais como na definição de estratégias de negociação de recursos com o governo federal, voltadas para garantir o suprimento de energia a longo prazo.

\section{CONSIDERAÇÕES FINAIS}

Este artigo analisou as interações energéticas (agregando-se as várias fontes de energia) entre o Estado de Minas Gerais e o restante do Brasil. Para isso, foi utilizado um modelo inter-regional de insumo-produto com incorporação de um setor de energia. O modelo foi construído a partir de uma tabela híbrida de insumo-produto regional, na qual as informações de vendas do setor de energia aos demais setores foram registradas em unidades físicas (tep) e não monetárias.

A aplicação feita neste trabalho permitiu identificar os setores mais relevantes dentro e fora do Estado de Minas Gerais para a demanda de energia que incide sobre o setor energético do Estado. Essa identificação foi feita a partir dos coeficientes de requerimento de energia intra e inter-regionais do modelo híbrido inter-regional e também com base nas relações de requerimentos diretos versus indiretos presentes na decomposição daqueles coeficientes. Dentro de Minas Gerais, os setores Ferro e Aço, Transporte, Energético e Outras Indústrias revelaram as maiores pressões de demanda sobre o setor de energia do Estado. Fora de Minas Gerais (i.e., no restante do Brasil), os setores Energético, Transporte, Outras Indústrias e Alimentos + Bebidas se mostraram mais importantes nesse sentido.

Dessa forma, a metodologia utilizada proporcionou informações relevantes para subsidiar a gestão e a formulação de políticas voltadas para garantir o suprimento adequado de energia por parte do planejador estadual. Do ponto de vista da gestão (curto e médio prazos) do suprimento de energia pelo Estado de Minas Gerais, os resultados indicam que um acompanhamento cuidadoso do comportamento conjuntural daquele grupo destacado de setores (dentro e fora do Estado) é relevante como estratégia de prevenção contra problemas de abastecimento de energia. Do ponto de vista do planejamento a longo prazo do fornecimento de energia pelo Estado, o desenho de políticas setoriais, de estratégias de negociação de recursos e ações com o governo federal podem se beneficiar com a aquisição de maior conhecimento sobre a estrutura setorial das pressões de demanda incidentes sobre o setor de energia em Minas Gerais.

Apesar de a metodologia ter permitido traçar um retrato relativamente refinado das interações energéticas entre Minas Gerais e o restante do Brasil, uma vez que foram analisados 14 setores de atividade em duas regiões espaciais, é possível fazê-la avançar bastante em termos de detalhamentos e desagregações, o que abre interessantes perspectivas para estudos futuros. Por exemplo, o setor de energia pode ser desagregado por tipo de energia produzida (petróleo, gás natural, eletricidade, carvão mineral e vegetal, etc.) e também pode-se incorporar um maior número de regiões/Estados no modelo inter-regional. Estes esforços de pesquisa podem ser frutíferos, proporcionando informa- 
ções mais relevantes tanto para os planejadores regionais/estaduais como para o próprio planejador nacional. Os ganhos decorrentes fornecerão subsídios de informação para uma gestão mais eficiente do suprimento de energia pela sociedade, com a conseqüente diminuição dos riscos de se comprometer o crescimento econômico.

\section{REFERÊNCIAS BIBLIOGRÁFICAS}

BDMG; FIPE/USP. Matriz inter-regional de insumo-produto para Minas Gerais/restante do Brasil. Belo Horizonte, 2002.

Bullard, C. W.; Herendeen, R. A. The energy cost of goods and services. Energy Policy, v. 3 n. 4, p. 268 278, 1975.

Casler, S. D.; Blair, P. D. Economic structure, fuel combustion, and pollution emissions, Ecological Economics, v. 22, p. 19-27, 1997.

CEMIG. Balanço energético do Estado de Minas Gerais, 2005. Disponível em: < http://www.cemig.com. $\mathrm{br}>$.

Gowdy, J. M.; Miller, J. L. Technological and demand change in energy use: an input-output analysis. Environment and Planning A, v. 19, n.10, p. 1387-1398, 1987.

Hilgemberg, E. M. Quantificação e efeitos econômicos do controle de emissões de co decorrentes do uso de gás natural, álcool e derivados de petróleo no Brasil: um modelo inter-regional de insumo-produto. 2004. Tese (Doutorado em Economia Aplicada), apresentada à Escola Superior de Agricultura Luiz de Queiroz / USP.

Hawdon, D.; Pearson, P. Input-output simulations of energy, environment, economy interactions in the UK. Energy Economics , v.17, n. 1, p. 73-86, 1995.

Isard, W. et al. Methods of interregional and regional analysis. New York: Ashgate, 1998.

Machado, V. M. Meio ambiente e comércio exterior: impactos da especialização comercial brasileira sobre o uso de energia e as emissões de carbono do país. 2002. Tese (Doutorado em Ciências em Planejamento Energético), apresentada a COPPE / UFRJ.

Miller, R. E.; Blair, P. D. Input-output analysis: foundations and extensions. New Jersey, Prentice Hall, 1985.

MME. Balanço energético nacional Ministério das Minas e Energia, 2005. Disponível em: < http://www. mme.gov.br>.

MME. Novo modelo do setor elétrico, 2004. Disponível em: <http://www.mme.gov.br>.

Torres, M. E. L.; Almeida. P. E. F. Cap. 2 - Minas Gerais do Século XXI. In: sustentando o desenvolvimento. BDMG, 2003.

Infra-estrutura:

Zhang, Z.; Folmer, H. Economic modelling approaches to cost estimates for the control of carbon dioxide emissions. Energy Economics v. 20, p. 101-120, 1998. 


\section{ANEXO - COMPATIBILIZAÇÃO DOS SETORES DO BEN E DA MATRIZ DE INSUMO- Produto}

\begin{tabular}{|l|l|}
\hline 1 Agropecuária & 10 Outras indústrias \\
1 Agropecuária & 23 Máquinas e equipamentos \\
2 Mineração e pelotização & 24 Material elétrico \\
2 Extrativa mineral & 25 Equipmentos eletrônicos \\
3 Petróleo e gás & 26 Automóveis, caminhões e ônibus \\
3 Minerais não metálicos & 27 Peças e outros veículos \\
4 Minerais não-metálicos & 28 Madeira e mobiliário \\
4 Ferro e aço & 29 Farmácia e veterinária \\
5 Siderurgia & 30 Artigos plásticos \\
5 Metais não ferrosos e outras metalurgias & 31 Indústrias diversas \\
6 Metalurgia dos não-ferrosos & 32 S.I.U.P \\
7 Outros metalúrgicos & 33 Construção civil \\
6 Papel e celulose & 34 Comunicações \\
8 Celulose papel e gráfica & 11 Comércio e serviços \\
9 Indústria da borracha & 35 Comércio \\
7 Química & 36 Instituições financeiras \\
10 Elementos químicos & 37 Serviços prestados à família \\
11 Refino do petróleo & 38 Serviços prestados à empresa \\
12 Químicos diversos & 39 Aluguel de imóveis \\
8 Alimentos e bebidas & 40 Serviços privados não mercantis \\
13 Indústria do café & 12 Transporte \\
14 Beneficiamento de produtos vegetais & 41 Transporte \\
15 Abate de animais & 13 Serviços públicos \\
16 Indústria de laticínios & 42 Administração pública \\
17 Fabricação de açúcar & 14 Setor energético \\
18 Fabricação de óleos vegetais & Lenha \\
19 Outros produtos alimentares & Petróleo e gás natural \\
9 Têxtil e vestuário & Extração de petróleo \\
20 Indústria têxtil & Refino de petróleo \\
21 Artigos de vestuário & Carvão e outros \\
22 Fabricação de calçados & Produtos energéticos de cana-de açúcar \\
& Eletricidade \\
& Resíduos \\
& \\
\hline
\end{tabular}

